\title{
Effect of zeta potentials on bovine serum albumin adsorption on crown composite resin surfaces in vitro
}

\author{
Nahoko Miyake ${ }^{1}$, Tadashi Miura ${ }^{2}$, Toru Sato $^{3}$, Masao Yoshinari $^{2}$ \\ ${ }^{1}$ Division of Prosthodontics, Department of Clinical Oral Health Science, Tokyo Dental College, Tokyo, Japan \\ ${ }^{2}$ Division of Oral Implants Research, Oral Health Science Center, Tokyo Dental College, Chiba, Japan \\ ${ }^{3}$ Department of Crown \& Bridge Prosthodontics, Tokyo Dental College, Chiba, Japan \\ Email: miyake@tdc.ac.jp
}

Received 1 January 2013; revised 1 March 2013; accepted 7 March 2013

\section{ABSTRACT}

We previously studied the mechanism underlying the adsorption of oral bacteria on the surfaces of dental prosthetic materials such as ceramics and resins in vitro. The aim of the present study was to examine bovine serum albumin (BSA) adsorption on crown composite resin surfaces by means of zeta potential. We measured the zeta potentials of resins alone, BSA alone, and resins after BSA adsorption. Eight resins were pulverized into powders $(300-1000 \mathrm{~nm})$. All experiments were conducted in $10 \mathrm{mM}$ sodium chloride solution (pH 6.5). BSA was dissolved in $10 \mathrm{mM}$ $\mathrm{NaCl}$ with a concentration of $2.0 \times 10^{-5} \mathrm{~mol} / \mathrm{l}$. An adsorption assay was performed for one hour at $37^{\circ} \mathrm{C}$ under continuous rotation $(6 \mathrm{rpm})$. The zeta potentials of both resins and BSA were negative, with BSA itself less negative than the resins themselves as an absolute value $(p<0.0001)$. The zeta potentials of seven resin surfaces after BSA adsorption were significantly less negative than were those of the resins without BSA adsorption $(p<0.0001)$. Eight resins were divided into two classes based on the size of the surface potential difference between each resin and the BSA. The difference in surface potential between the resins and the BSA were small, leading to the theory that particles with identical charges repulse each other, and the amounts of adsorbed BSA on these resins might be less. On the other, when the differences between the other resins and BSA are large, so that the repulsive force between two nonidentical particles becomes zero and an attractive force might be generated, then more BSA might be adsorbed on those resins. Therefore, the zeta potentials were affected by BSA adsorption and became less negative. These results suggested that electrostatic interactions play an important role in the adsorption of BSA on resin surfaces.

Keywords: Zeta Potential; Crown Composite Resins;
Bovine Serum Albumin; Adsorption; Electrostatic Interactions

\section{INTRODUCTION}

Forces affecting the adsorption, desorption, and other behavioral characteristics of microorganisms, proteins, and other substances at interfaces can be broadly classified into two types: 1) forces acting between molecules making up a substance (hydrophobicity), and 2) electrical interactions resulting from surface charges [1]. The hydrophobicity of substance surfaces can be qualitatively evaluated by measuring contact angles and surface free energy. However, surface potentials are difficult to measure. Therefore, surface electrokinetic potentials (zeta potentials) are calculated and quantified on the basis of electrophoretic mobility. Changes in surface charge density can be determined by measuring zeta potentials before and after adsorption, thereby enabling the calculation of adsorption capacity. In the development of materials for artificial organs, this principle has been used to evaluate the adsorption behavior of proteins, enzymes, and other biological macromolecules, to study the effects of washing on protein removal, and to investigate the prevention of adsorption by coating [2].

We previously studied the mechanism underlying the adsorption of oral bacteria on the surfaces of prosthetic materials. Our results showed that electrostatic interactions have a role in the adsorption of oral bacteria on the surfaces of prosthetic materials such as ceramics and resins. In addition, zeta potentials were demonstrated to be important factors in the adsorption of oral bacteria on prosthetic materials [3]. We then examined the adsorption of bovine serum albumin (BSA) on hydroxyapatite surfaces used as a blocking agent of bacteria adsorption on experimental salivary pellicles [4]. The results suggested that BSA adsorption on hydroxyapatite surfaces is related to electrostatic interaction [5].

The aim of the present study was to employ zeta potential to examine BSA adsorption on crown composite 
resin (resin) surfaces. We measured zeta potentials of the resins alone, the BSA alone, and the resin surfaces after BSA adsorption. Based on the zeta potentials obtained, we discuss how the electrostatic interactions are involved in the adsorption of BSA on resin surfaces.

\section{MATERIALS AND METHODS}

\subsection{Resin Materials}

Material specimens were prepared from eight crown composite resins (Table 1): Ceramage (Shofu, Kyoto, Japan), Epricord (Kuraray Noritake Dental, Kurashiki, Japan), Estenia C\&B (Kuraray Noritake Dental, Kurashiki, Japan), Gradia (GC Dental Products, Kasugai, Japan), Gradia Forte (GC Dental Products, Kasugai, Japan), Luna-wing (Yamamoto Precious Metal, Osaka, Japan), Prossimo (GC Dental Products, Kasugai, Japan), and Solidex (Shofu, Kyoto, Japan). The resins were polymerized according to the manufacturer's instructions and were then pulverized into powder with a particle diameter of $300-1000 \mathrm{~nm}$.

\subsection{BSA}

BSA (Sigma-Aldrich Co., St. Louis, Missouri, USA) was used as a blocking agent to the resin materials.

\subsection{Monitor Particles}

Polystyrene latex spheres (particle diameter, $520 \mathrm{~nm}$, Otsuka Electronics Co., Ltd.) coated with hydroxypropyl cellulose $\left(\mathrm{MV}=30 \times 10^{5}\right.$, Scientific Polymer Products Inc., Ontario, New York, USA), were used as monitor particles. They were measured with each material type to confirm that the equipment was operating properly [6].

\subsection{Measurement of Zeta Potentials}

The electrophoretic mobilities were measured using a zeta potential analyzer (ZEECOM ZC-2000 ${ }^{\mathrm{TM}}$, Microtec, Chiba, Japan). All experiments were conducted in 10 $\mathrm{mM}$ sodium chloride solution $(\mathrm{NaCl})$ at $\mathrm{pH}$ 6.5. BSA dissolved in $10 \mathrm{mM} \mathrm{NaCl}$ was concentrated to $2.0 \times 10^{-5}$ $\mathrm{mol} / \mathrm{l}$. Adsorption experiments were performed for one hour at $37^{\circ} \mathrm{C}$ under continuous rotation at $6 \mathrm{rpm}$. Zeta potentials before adsorption were compared with the values after adsorption.

\subsection{Statistical Analysis}

Experimental data were expressed as mean \pm SD. Students T-test was used to determine significance, and $p$ values of 0.05 or less were considered significant.

\section{RESULTS AND DISCUSSION}

The zeta potentials of all of the resins, Gradia Forte $(-48.1 \pm 8.4 \mathrm{mV})$, Ceramage $(-42.3 \pm 7.4 \mathrm{mV})$, Gradia $(-40.8 \pm 10.1 \mathrm{mV})$, Luna-wing $(-38.1 \pm 9.0 \mathrm{mV})$, Estenia $\mathrm{C} \& \mathrm{~B}(-33.5 \pm 7.9 \mathrm{mV})$, Prossimo $(-32.5 \pm 6.4 \mathrm{mV})$, Epricord $(-31.7 \pm 6.0 \mathrm{mV})$, and Solidex $(-29.9 \pm 7.4 \mathrm{mV})$ were negative. The zero potential of BSA $(-19.6 \pm 6.0$ $\mathrm{mV}$ ) was negative with BSA itself less negative than were those of the resins as an absolute value $(\mathrm{p}<0.0001)$ (Table 2).

The zeta potentials of the resin surfaces after BSA ad-

Table 1. The component of used resin materials in this study.

\begin{tabular}{|c|c|c|}
\hline Brand name & Manufacture & Component \\
\hline Ceramage & Shofu & UDMA, urethane diacrylate, zirconium silicate, colorant \\
\hline Epricord & Kuraray Noritake Dental & $\begin{array}{l}\text { urethane-based tetra-methacrylate, TEGDMA, other methacrylate ester } \\
\text { surface-treated organic composite filler, surface-treated powdered glass } \\
\text { surface-treated silica-based microfiller, photopolymerization catalyst, colorant }\end{array}$ \\
\hline Estenia C\&B & Kuraray Noritake Dental & $\begin{array}{l}\text { urethane-based methacryl monomer, methacrylic acid-based monomer } \\
\text { surface-treated powdered glass, surface-treated alumina-based microfiller } \\
\text { photopolymerization catalyst, colorant }\end{array}$ \\
\hline Gradia & GC Dental Products & $\begin{array}{l}\text { amino-silicate glass, amorphous precipitated silica } \\
\text { di-2-methacryloyloxyethyl 2,2,4-trimethylhexamethylene dicarbamate } \\
\text { neopentylglycol dimethacrylate }\end{array}$ \\
\hline Gradia Forte & GC Dental Products & $\begin{array}{l}\text { amino-silicate glass, amorphous precipitated silica, } \\
\text { di-2-methacryloyloxyethyl 2,2,4-trimethylhexamethylene dicarbamate } \\
\text { neopentylglycol dimethacrylate }\end{array}$ \\
\hline Luna-wing & Yamamoto Precious Metal & methacryl-based monomer, organic compound filler, inorganic filler, colorants \\
\hline Prossimo & GC Dental Products & $\begin{array}{l}\text { Organic-inorganic composite filler, urethane-based dimethacrylate } \\
\text { silica fine powder, glass powder }\end{array}$ \\
\hline Solidex & Shofu & UDMA, organic fillers, silica powder, colorants \\
\hline
\end{tabular}


sorption were: Solidex $(-34.6 \pm 6.8 \mathrm{mV})$, Gradia Forte $(-33.9 \pm 4.1 \mathrm{mV})$, Estenia C\&B $(-28.2 \pm 7.9 \mathrm{mV})$, Ceramage $(-25.9 \pm 5.6 \mathrm{mV})$, Epricord $(-25.1 \pm 5.4 \mathrm{mV})$, Gradia $(-21.2 \pm 5.2 \mathrm{mV})$, Luna-wing $(-21.2 \pm 5.5 \mathrm{mV})$, and Prossimo $(-21.0 \pm 5.0 \mathrm{mV}$ ) (Table 3). The zeta potentials of the seven resin surfaces after BSA adsorption were significantly less negative than were those of the resins without BSA adsorption $(\mathrm{p}<0.0001)$.

Previous studies have used zeta potentials to examine the adsorption of saliva proteins and oral bacteria in detail [7-10]. Most studies used saliva or several proteins, such as BSA, human serum albumin, immunoglobulin G, fibrinogen, lysozyme, and $\alpha$-lactalbumin. In a study by Young et al. [10], the zeta potentials of human enamel and hydroxyapatite particles became less negative after adsorption of parotid saliva and whole saliva. However,

Table 2. The zeta potential of resin-surfaces.

\begin{tabular}{cc}
\hline & Zeta potential $(\mathrm{mV})$ \\
\hline Ceramage & $-42.3 \pm 7.4^{*}$ \\
Epricord & $-31.7 \pm 6.0^{*}$ \\
Estenia C\&B & $-33.5 \pm 7.9^{*}$ \\
Gradia & $-40.8 \pm 10.1^{*}$ \\
Gradia Forte & $-48.1 \pm 8.4^{*}$ \\
Luna-wing & $-38.1 \pm 9.0^{*}$ \\
Prossimo & $-32.5 \pm 6.4^{*}$ \\
Solidex & $-29.9 \pm 7.4^{*}$ \\
BSA & $-19.6 \pm 6.0$
\end{tabular}

Values are the mean $\pm \mathrm{SD}$ of zeta potential of resin-surfaces from 150 subjects; The zeta potentials of both resins and BSA were negative. Asterisks indicate significant difference with BSA itself less negative than resins themselves as an absolute value $(\mathrm{p}<0.0001)$.

Table 3. The zeta potential of resin-surfaces after BSA adsorption.

\begin{tabular}{cc}
\hline & Zeta potential $(\mathrm{mV})$ \\
\hline Ceramage & $-25.9 \pm 5.6^{*}$ \\
Epricord & $-25.1 \pm 5.4^{*}$ \\
Estenia C\&B & $-28.2 \pm 5.7^{*}$ \\
Gradia & $-21.2 \pm 5.2^{*}$ \\
Gradia Forte & $-33.9 \pm 4.1^{*}$ \\
Luna-wing & $-21.2 \pm 5.5^{*}$ \\
Prossimo & $-21.0 \pm 5.0^{*}$ \\
Solidex & $-34.6 \pm 6.8$ \\
\hline
\end{tabular}

Values are the mean $\pm \mathrm{SD}$ of zeta potential of resin-surfaces after BSA adsorption from 150 subjects. Asterisks indicate the zeta potentials after BSA adsorption were significantly less negative than those of resins without BSA adsorption $(\mathrm{p}<0.0001)$. those studies did not measure the zeta potentials of the proteins used. In addition, they did not clarify whether protein adsorption was caused by electrostatic interactions or by hydrophobicity. Longsworth [11] estimated that the isoelectric point of BSA is $\mathrm{pH}_{0}=-4.89$ by measuring the zeta potential of BSA. A similar value was obtained in this study even though a different solvent was used.

In our study, the eight resins were divided into two classes from the size of the surface potential difference between the resins and BSA. The difference between the resins, such as Estenia C\&B, Epricord, Prossimo, and Solidex, and BSA was small, leading to the theory that particles with identical charges repulse each other [12], and the amounts of adsorbed BSA on these resins might be less. On the other hand, when the difference of the other resins, such as Ceramage, Gradia, Gradia Forte, and Luna-wing, and BSA was large so that the repulsive force between two nonidentical particles becomes zero and an attractive force might be generated [12], then more BSA might be adsorbed on those resins. Therefore, the zeta potentials were affected by BSA adsorption and became less negative.

These results suggested that the zeta potentials of resins with BSA adsorption were significantly less negative than were those of resins without BSA adsorption and that electrostatic interactions play an important role in BSA adsorption on resin surfaces.

Morisaki [13] found that the cell surface of bacteria isolated from a soil environment is negatively charged. They also found that bacterial strains with slower proliferation rates have smaller absolute surface charge values, higher hydrophobicity, and stronger adhesive capacity. A previous study attempted to investigate the adhesion of oral bacteria to dental restorative materials, focusing on surface free energy [14].

Further studies are needed to evaluate the hydrophobicity of substance surfaces by measuring contact angles and surface free energy. Studies are also needed in the development of prosthetic materials by evaluating the adsorption of proteins, enzymes, and other biological macromolecules on the surfaces of prosthetic materials and by studying the removal of proteins by washing and the prevention of adsorption by coating.

\section{CONCLUSIONS}

We measured the zeta potentials of resins alone, BSA alone, and different resin surfaces after BSA adsorption in vitro. The following conclusions were reached based on electrostatic interactions. The zeta potentials of both the resins and BSA were negative, with BSA itself less negative than the resins as an absolute value $(\mathrm{p}<0.0001)$. The zeta potentials of seven resin surfaces after BSA 
adsorption were significantly less negative than were those of resins without BSA adsorption $(\mathrm{p}<0.0001)$.

These results suggested that the zeta potentials of resins with BSA adsorption were significantly less negative than were those of resins without BSA adsorption. Electrostatic interactions play an important role in the adsorption of BSA on resin surfaces.

\section{ACKNOWLEDGEMENTS}

This study was supported by a Grant-in-Aid for Scientific Research of (C) (No. 20592281). The authors thank Dr. Yumiko Tomita, previously belonging to the Department of Biochemistry of Tokyo Dental College, for her guidance and support.

\section{REFERENCES}

[1] Kasahara, Y. and Nigaki, T. (1998) Surface characteristic of bacteria. In: Morisaki, H., Ohshima, H. and Isobe, K. Eds., Biofilm, Science Forum, Tokyo, 72-74.

[2] Furusawa, K. (1995) Adsorption and zeta potential. In: Kitahara, F., Furusawa, K., Ozaki, M. and Ohshima, H. Eds., Zeta Potential, Scientist, Tokyo, 114-132.

[3] Nakamura, N. (1995) Oral bacterial adsorption to the materials for prosthodontics in vitro. Shikwa Gakuho, 95, 375-390.

[4] Gibbons, R.J. and Etherden, I. (1985) Albumin as a blocking agent in studies of streptococcal adsorption to experimental salivary pellicles. Infection and Immunity, 50, 592-594.

[5] Miyake, N., Sato, T. and Maki, Y. (2010) Effect zeta potential on bovine serum albumin adsorption to hydroxyapatite surface in vitro. Shikwa Gakuho, 110, 105-109.

[6] Miura, T., Miyake, N., Tanabe, K. and Yoshinari, M. (2011) Change in zeta potential with physicochemical treatment of surface of anatase-form tinania particles. Journal of Oral Tissue Engineering, 9, 64-70.
[7] Weerkamp, A.H., Uyen, H.M. and Busscher, H. J. (1988) Effect of zeta potential and surface energy on bacterial adhesion to uncoated and saliva-coated human enamel and dentin. Journal of Dental Research, 67, 1483-1487. doi:10.1177/00220345880670120801

[8] Van der Mei, H.C., Meijer, S. and Busscher, H. J. (1998) Electrophoretic mobilities of protein-coated Hexadecane droplets at different $\mathrm{pH}$. Journal of Colloid and Interface Science, 205, 185-190. doi:10.1006/jcis. 1998.5669

[9] Kambara, M. and Norde, W. (1995) Influence of fluoride applications on some physicochemical surface properties of synthetic hydroxyapatite and human dental enamel and its consequences for protein adsorption. Caries Research, 29, 210-217. doi: $10.1159 / 000262071$

[10] Young, A., Smistad, G., Karlsen, J., Rölla, G. and Rykke, M. (1997) Zeta potentials of human enamel and crown composite resins as measured by the coulter DELSA 440 . Advances in Dental Research, 11, 560-565. doi:10.1177/08959374970110042501

[11] Longsworth, L.G. and Jacobsen, C.F. (1949) An electrophoretic study of the binding of salt ions by beta-lactoglobulin and bovine serum albumin. Journal of Physical Chemistry, 53, 126-135. doi:10.1021/j150466a010

[12] Bierman, A. (1955) Electrostatic forces between nonidentical colloidal particles. Journal of Colloid Science, 10, 231-245. doi:10.1016/0095-8522(55)90036-2

[13] Morisaki, H. (2009) Surface characteristics of bacterial cells living in natural environments and its relevance with bacterial survival strategy. Proceedings of the 48th Annual Meeting of Japan Oil Chemists' Society, Nagoya, 10-12 September 2009, 86-87.

[14] Weerkamp, A.H., Quirynen, M., Marechal, M., Van der Mei, H.C., Van Steenberghe, D. and Busscher, H. J. (1989) The role of surface free energy in vivo formation of dental plaque on human enamel and polymeric substrata. Microbial Ecology in Health and Disease, 2, 11-18. doi:10.3109/08910608909140196 